\title{
Article \\ Therapeutic Options for Recurrent Glioblastoma-Efficacy of Talaporfin Sodium Mediated Photodynamic Therapy
}

\author{
Tatsuya Kobayashi ${ }^{1}{ }^{(}$, , Masayuki Nitta ${ }^{1,2, *}$, Kazuhide Shimizu $^{3,4}$, Taiichi Saito ${ }^{1,2}{ }^{\oplus}$, Shunsuke Tsuzuki ${ }^{1}$, \\ Atsushi Fukui ${ }^{1}$, Shunichi Koriyama ${ }^{1}$, Atsushi Kuwano ${ }^{1}$, Takashi Komori ${ }^{5}{ }^{\circledR}$, Kenta Masui ${ }^{6}{ }^{(}$, \\ Taketoshi Maehara ${ }^{4}$, Takakazu Kawamata ${ }^{1}$ and Yoshihiro Muragaki ${ }^{1,2}$
}

\section{check for} updates

Citation: Kobayashi, T.; Nitta, M. Shimizu, K.; Saito, T.; Tsuzuki, S.; Fukui, A.; Koriyama, S.; Kuwano, A.; Komori, T.; Masui, K.; et al. Therapeutic Options for Recurrent Glioblastoma-Efficacy of Talaporfin Sodium Mediated Photodynamic Therapy. Pharmaceutics 2022, 14, 353. https://doi.org/10.3390/ pharmaceutics14020353

Academic Editor: Giuseppina Ioele

Received: 22 December 2021

Accepted: 1 February 2022

Published: 2 February 2022

Publisher's Note: MDPI stays neutral with regard to jurisdictional claims in published maps and institutional affiliations.

Copyright: (C) 2022 by the authors. Licensee MDPI, Basel, Switzerland. This article is an open access article distributed under the terms and conditions of the Creative Commons Attribution (CC BY) license (https:// creativecommons.org/licenses/by/ $4.0 /)$.
1 Department of Neurosurgery, Tokyo Women's Medical University, 8-1 Kawadacho, Shinjuku-ku, Tokyo 162-8666, Japan; opera58840428@gmail.com (T.K.); taiichis@gmail.com (T.S.); st09212005@yahoo.co.jp (S.T.); fukui.atsushi@twmu.ac.jp (A.F.); song.4.u.01.16@gmail.com (S.K.); cho_hunseki@yahoo.co.jp (A.K.); kawamata.takakazu@twmu.ac.jp (T.K.); ymuragaki@twmu.ac.jp (Y.M.)

2 Faculty of Advanced Techno-Surgery, Tokyo Women's Medical University, 8-1 Kawadacho, Shinjuku-ku, Tokyo 162-8666, Japan

3 Department of Neurosurgery, Massachusetts General Hospital, Harvard Medical School, 185 Cambridge Street, Boston, MA 02114, USA; KSHIMIZU3@mgh.harvard.edu

4 Department of Neurosurgery, Tokyo Medical and Dental University, 1-5-45 Yushima, Bunkyo-ku, Tokyo 113-8519, Japan; maehara.nsrg@tmd.ac.jp

5 Department of Laboratory Medicine and Pathology (Neuropathology), Tokyo Metropolitan Neurological Hospital, 2-6-1 Musashidai, Fuchu-shi, Tokyo 183-0042, Japan; komori-tk@igakuken.or.jp

6 Department of Pathology, Tokyo Women's Medical University, 8-1 Kawadacho, Shinjuku-ku, Tokyo 162-8666, Japan; masui-kn@twmu.ac.jp

* Correspondence: nitta.masayuki@twmu.ac.jp

\begin{abstract}
Recurrent glioblastoma (GBM) remains one of the most challenging clinical issues, with no standard treatment and effective treatment options. To evaluate the efficacy of talaporfin sodium (TS) mediated photodynamic therapy (PDT) as a new treatment for this condition, we retrospectively analyzed 70 patients who underwent surgery with PDT (PDT group) for recurrent GBM and 38 patients who underwent surgery alone (control group). The median progression-free survival (PFS) in the PDT and control groups after second surgery was 5.7 and 2.2 months, respectively $(p=0.0043)$. The median overall survival (OS) after the second surgery was 16.0 and 12.8 months, respectively $(p=0.031)$. Both univariate and multivariate analyses indicated that surgery with PDT and a preoperative Karnofsky Performance Scale were significant independent prognostic factors for PFS and OS. In the PDT group, there was no significant difference regarding PFS and OS between patients whose previous pathology before recurrence was already GBM and those who had malignant transformation to GBM from lower grade glioma. There was also no significant difference in TS accumulation in the tumor between these two groups. According to these results, additional PDT treatment for recurrent GBM could have potential survival benefits and its efficacy is independent of the pre-recurrence pathology.
\end{abstract}

Keywords: recurrent glioblastoma; photodynamic therapy; talaporfin sodium; photosensitizer; lower grade glioma

\section{Introduction}

Glioblastoma (GBM) is one of the most malignant primary brain tumors with a quite poor prognosis. The standard treatment for newly diagnosed GBM is maximal surgical resection followed by radiotherapy with concomitant and adjuvant temozolomide-based chemotherapy. Nevertheless, the median survival time for patients who complete the standard treatment is only about 15 months or less [1]. One factor that contributes to the unsatisfactory prognosis in GBM is that most recur following standard treatment. On recurrence, 
the therapeutic options include surgical rechallenge, additional existing chemotherapy, and additional radiation, but their efficacy is limited. The median overall survival (OS) after various retreatments for recurrent GBM has been reported at 6.5-7.6 months [2-4]. There is currently no standard treatment for recurrent GBM, because no existing therapy has demonstrated superiority $[3,5,6]$. Therefore, new effective treatments for this condition are urgently required.

Photodynamic therapy (PDT) uses specific wavelengths of light to activate photosensitizers accumulated in the tumor. When the photosensitizer is activated, it generates reactive oxygen species (ROS) in the local irradiated area. The ROS destroy various cellular organelles and tumor blood vessels, thereby destroying the tumor itself [7]. There are several types of photosensitizers, each with different biological and pharmacokinetic characteristics, including subcellular localization, excitation and emission wavelength, the mechanism for inducing cell death, and the uptake or clearance level of the specific tissue $[8,9]$.

Recently in Japan, talaporfin sodium (mono-L-aspartyl chlorine, NPe6, TS), a chlorinbased photosensitizer has been clinically applied in PDT for lung and esophageal cancers, and in malignant brain tumors [10-12]. In a phase II trial of talaporfin sodium mediated PDT (TS-PDT) for malignant brain tumors, the median progression-free survival (PFS) and the median OS for newly diagnosed GBM were 12.0 and 24.8 months, respectively [12]. Furthermore, we recently performed a retrospective analysis of the efficacy of intraoperative TS-PDT for newly diagnosed GBM and reported that the TS-PDT group had a significantly better PFS of 19.6 months and OS of 27.4 months compared with standard treatment [13]. Consideration of these favorable results led TS-PDT to be substantiated as a valuable additional treatment for newly diagnosed GBM. On the other hand, there are no cohort or case-control studies of TS-PDT for recurrent GBM, and its efficacy is still unclear.

Given these current circumstances, as the second report on the clinical efficacy of TS-PDT for a malignant brain tumor, we analyzed the prognostic data of single-center experience cases and evaluated the therapeutic effect on recurrent GBM in this study. To analyze tumor pathology and pharmacokinetics of TS, we also examined the amount of TS uptake in recurrent glioblastoma and verified whether the effect of TS-PDT depended on previous pathological results before recurrence.

\section{Materials and Methods}

\subsection{Patient Selection and Treatment Criteria}

In this single-center retrospective analysis, 70 consecutive patients who underwent surgical resection and intraoperative TS-PDT (PDT group) for recurrent malignant glioma between February 2014 and December 2018 were compared with 38 consecutive patients with recurrent GBM who underwent surgical resection alone (control group) during the same period. At our institution, patients with a Karnofsky Performance Status (KPS) score $\geq 60$ were considered for reoperation if they developed recurrence of malignant glioma after standard multidisciplinary treatment. The indication for surgery was restricted to cases in which total resection of the recurrent contrast-enhancing lesion was considered feasible. For patients with a KPS score of 40 or 50 and a strong desire for surgery, the surgical indication was limited to those expected to experience an improvement in clinical symptoms with reoperation in addition to the previously stated conditions. This study was approved by the Institutional Review Board of our institution (approval code: 3540-R6).

\subsection{Intraoperative TS-PDT Protocol}

Intraoperative TS-PDT for recurrent GBM was performed using the same protocol that we previously reported for newly diagnosed GBM and other malignant brain tumors in Japan [12-14]. Patients received a single intravenous injection of TS at a dose of $40 \mathrm{mg} / \mathrm{m}^{2}, 22-26 \mathrm{~h}$ before surgery. After maximal resection of the contrast-enhanced lesions, laser irradiation to the resection cavity was performed using a $664 \mathrm{~nm}$ semiconductor laser (PD laser BT, Meiji Seika Co., Ltd., Tokyo, Japan) with an irradiation power density of 
$150 \mathrm{~mW} / \mathrm{cm}^{2}$ and an irradiation energy density of $27 \mathrm{~J} / \mathrm{cm}^{2}$ within a circle (diameter: $1.5 \mathrm{~cm}$ ) per location. The irradiation was performed to cover the entire resection cavity without overlapping of the irradiation fields.

\subsection{Neuropathological Analysis}

Histopathological diagnosis was conducted based on the WHO guidelines of 2007 and 2016 [15,16]. For patients diagnosed according to the 2007 WHO classification, IDH mutation was retrospectively analyzed and re-diagnosed using the 2016 WHO classification. IDH mutation status was examined by immunohistochemistry using R132H-specific antibody (DIA-H09, Dianova GmbH, Hamburg, Germany). In case of negative results, direct DNA sequencing of the tumor sample was additionally performed. Mib-1 index was assessed using immunohistochemistry with Mib-1 monoclonal antibody (M7240, Agilent Technologies, Santa Clara, CA, USA). The presence of $1 \mathrm{p} / 19 \mathrm{q}$ codeletion was analyzed using fluorescence in situ hybridization. The methylation status of O-6-methylguanineDNA methyltransferase (MGMT) was not evaluated, but alternatively, the expression of MGMT protein was determined by conducting immunohistochemistry with anti-MGMT monoclonal antibody (MAB16200, Merck, Darmstadt, Germany).

\subsection{Evaluation of TS Uptake in Recurrent GBM}

TS exhibits a soret absorption band at $\sim 400 \mathrm{~nm}$ and produces emission at a wavelength light of $\sim 660 \mathrm{~nm}$ after excitation [17]. A fluorescence microscope (BZ-X710, KEYENCE, Osaka, Japan) at an excitation wavelength of $400 \mathrm{~nm}$ to image and evaluate the uptake of TS in recurrent GBM samples. To quantify the TS uptake in recurrent GBM, we measured the peak fluorescence intensity from each tumor sample using a semiconductor laser unit (LDS1005BL, Precise Gauges Co., Ltd., Shizuoka, Japan) according to a previously reported method [18] and compared the relationship between TS uptake and previous pathology before recurrence.

\subsection{Patient Assessment and Follow-Up}

All patients were evaluated with 0.4T intraoperative MRI images (APERTO Lucent, Hitachi, Ltd., Tokyo, Japan) before and after tumor removal or 1.5T MRI images during the early postoperative period (within $72 \mathrm{~h}$ after surgery). Based on these MRI images, the extent of resection (EOR) of the contrast-enhanced lesion was categorized as follows: Gross total resection (GTR) was considered for an EOR > 98\%, subtotal resection (STR) 95-98\%, and partial resection $(\mathrm{PR})<95 \%$. An additional postoperative MRI was performed 2 weeks after surgery, followed by imaging every month; assessment of tumor recurrence was determined based on the Response Assessment in Neuro-oncology (RANO) criteria [19]. The severity of adverse events was determined based on the Common Terminology Criteria for Adverse Events (CTCAE) version 5.0.

\subsection{Statistical Analysis}

All statistical analyses were performed using EZR version 1.54 (Saitama Medical Center, Jichi Medical University, Saitama, Japan) [20], which is a graphical user interface for R (The R Foundation for Statistical Computing, Vienna, Austria).

For intergroup comparison, the Mann-Whitney $U$ test was used for continuous variables and the $\chi^{2}$ test for categorical variables. Time-to-event analysis was performed using Kaplan-Meier curves and log-rank tests. PFS was defined as the time from the date of operation for recurrent GBM to the date of documented evidence of tumor progression according to the RANO criteria. OS was defined as the time from the date of surgery for recurrent GBM to the date of death or censoring at the last known date alive. Univariate and multivariate analyses were performed using the Cox proportional hazards model. Statistical significance was set at a $p$ value $<0.05$ and all reported $p$-values are two-sided. 


\section{Results}

\subsection{Patient Demographics and Characteristics}

The demographics and clinicopathological characteristics of patients in the PDT and control groups were described in Table 1 . Among the 108 patients, the PDT group comprised 70 patients (male 56\%; female $44 \%$ ), with the median age at reoperation being 43.5 (range 20-80) years. The control group contained 38 patients (male 68\%; female $32 \%$ ), with a median age at reoperation of 42 (range 16-71) years. There were no significant differences regarding age and sex between the two groups ( $p=0.32$ and 0.22 , respectively), and there was no significant difference in preoperative KPS $(p=0.063)$. In the PDT group, results of the EOR evaluation were GTR 91.4\%, STR 5.7\%, PR 2.9\%, and in the control group they were GTR 94.7\%, PR 5.3\% ( $p=0.37)$. The histopathological results revealed 69 cases of GBM and one case of gliosarcoma in the PDT group, and all 38 cases were GBM in the control group. Of the 69 patients in the PDT group with GBM, 43 had already been previously diagnosed with GBM based on pathology before recurrence, and 26 had been diagnosed as lower grade glioma (LGG). IDH1 R132H mutation was identified in $22.9 \%$ of the PDT group and $39.5 \%$ of the control group $(p=0.11)$. The median Mib-1 index in the PDT and control groups were 17.0 (range 1.6-51.4) and 20.7 (range 4.0-46.8), respectively, with no significant difference $(p=0.11)$. There was no $1 p / 19 q$ codeletion in any patient, and there was no difference in O-6-Methylguanine-DNA methyltransferase (MGMT) protein expression between the two groups $(p=0.73)$.

Table 1. Patient demographics and characteristics.

\begin{tabular}{|c|c|c|c|}
\hline & PDT & Control & $p$ Value \\
\hline No. of patients & 70 & 38 & \\
\hline \multicolumn{4}{|l|}{ Age } \\
\hline Average (year) $\pm S D$ & $46.7 \pm 13.3$ & $43.4 \pm 13.3$ & 0.32 \\
\hline Median (range) & $43.5(20-80)$ & $42(16-71)$ & \\
\hline Sex & & & 0.22 \\
\hline Male & $39(56 \%)$ & $26(68 \%)$ & \\
\hline Female & $31(44 \%)$ & $12(32 \%)$ & \\
\hline \multicolumn{4}{|l|}{ Median preoperative KPS } \\
\hline KPS score (range) & $80(40-90)$ & $85(50-100)$ & 0.063 \\
\hline EOR & & & 0.37 \\
\hline GTR & $64(91.4 \%)$ & $36(94.7 \%)$ & \\
\hline STR & $4(5.7 \%)$ & 0 & \\
\hline PR & $2(2.9 \%)$ & $2(5.3 \%)$ & \\
\hline Histopathology of rec. & & & 1 \\
\hline GBM & $69(98.6 \%)$ & $38(100 \%)$ & \\
\hline Gliosarcoma & $1(1.4 \%)$ & 0 & \\
\hline \multicolumn{4}{|c|}{ Previous pathology before rec. } \\
\hline GBM & $43(62.3 \%)$ & $16(42.1 \%)$ & 0.071 \\
\hline LGG & $26(37.7 \%)$ & $22(57.9 \%)$ & \\
\hline \multicolumn{4}{|l|}{ IDH mutation } \\
\hline Rate of IDH mutation & $22.9 \%(16 / 70)$ & $39.5 \%(15 / 38)$ & 0.11 \\
\hline \multicolumn{4}{|l|}{ Mib-1 } \\
\hline Average \pm SD & $19.8 \pm 12.0$ & $23.4 \pm 11.4$ & 0.11 \\
\hline Median (range) & $17.0(1.6-51.4)$ & $20.7(4.0-46.8)$ & \\
\hline MGMT protein expression & & & 0.73 \\
\hline High & $15(23.1 \%)$ & $9(24.3 \%)$ & \\
\hline Low & $28(43.1 \%)$ & $13(35.1 \%)$ & \\
\hline None & $22(33.8 \%)$ & $15(40.5 \%)$ & \\
\hline
\end{tabular}




\subsection{Patient Safety}

The complication rate was 4.3\% (3 patients) in the PDT group and $0 \%$ (0 patients) in the control group $(p=0.55)$. In the PDT group, one patient experienced wound dehiscence (grade 3) and required surgical reconstruction, one patient had cerebrospinal fluid leakage (grade 2), and one patient had acute epidural hematoma as postoperative hemorrhage (grade 3 ) and required surgical treatment. No other adverse events $\geq$ grade 3 according to the CTCAE version 5.0, were observed in both groups.

\subsection{Survival Analysis}

The median PFS after surgery for recurrence of the 70 patients in the PDT group was 5.7 months (95\% confidence interval [CI] 3.4-7.1), and the median PFS in the control group was 2.2 months (95\% CI 1.5-4.0); the PDT group exhibited significantly longer PFS than the control group ( $p=0.0043$, Figure 1A). The median OS after surgery for recurrence in the PDT group was 16.0 (95\% CI 13.7-22.5) months, the 1-year OS rate was 73\% and the 2-year OS rate was $37.4 \%$, whereas the median OS in the control group was 12.8 (95\% CI 9.3-15.0) months, the 1-year OS rate was $58.8 \%$, and the 2-year OS rate was $11.5 \%$; the PDT group exhibited better OS than the control group which was statistically significant $(p=0.031$, Figure 1B).

A

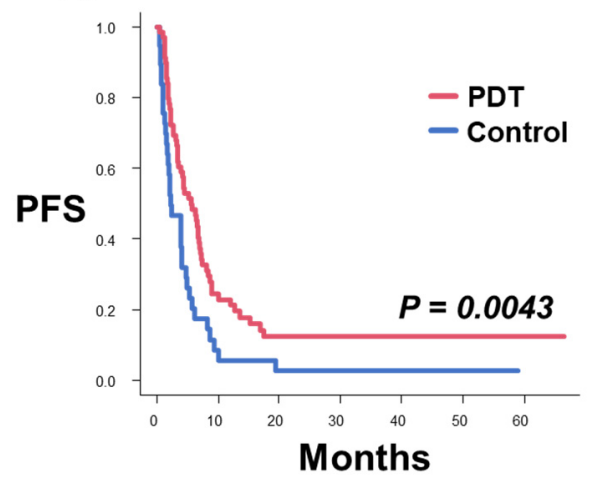

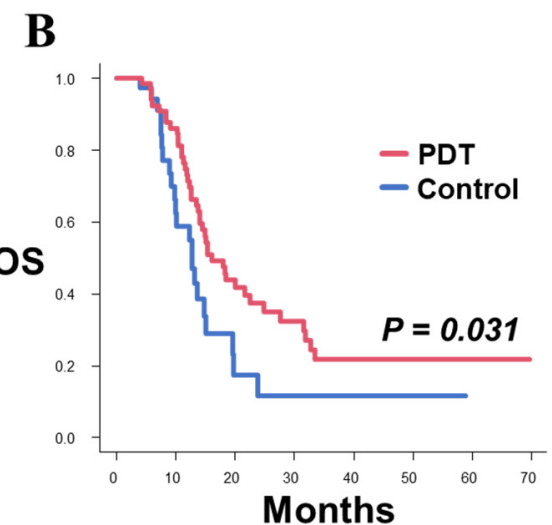

Figure 1. Kaplan-Meier survival curves for PFS and OS after surgery for recurrence in the PDT and control groups. (A) Patients in the PDT group showed significantly longer PFS than the patients in the control group (median PFS: PDT 5.7 months, control 2.2 months; $p=0.0043$ ). (B) Patients in the PDT group showed significantly longer OS than the patients in the control group (median OS: PDT 16.0 months, Control 12.8 months; $p=0.031$ ).

In the PDT group, there were 43 patients whose previous pathology before recurrence was GBM (GBM group), and 26 patients whose previous pathology before recurrence was lower-grade glioma (LGG group). The median PFS after recurrence in the GBM and LGG groups were 6.3 (95\% CI 3.1-8.4) and 4.2 (95\% CI 2.7-6.9) months, respectively ( $p=0.31$, Figure 2A). The median OS after recurrence in the GBM and LGG groups were 15.4 (95\% CI 13.4-31.6) and 18.3 (95\% CI 11.9-33.4) months, respectively ( $p=0.91$, Figure 2B). Therefore, PFS and OS exhibited no significant between-group differences.

\subsection{Univariate and Multivariate Analysis}

The relationship between PFS, OS, and prognostic factors such as age, preoperative KPS score, pre-recurrence pathology, IDH mutation, and addition of PDT were examined in univariate and multivariate analyses. The results revealed that preoperative KPS score and the addition of PDT were independent and significant prognostic factors in both univariate and multivariate analyses for PFS (Table 2). Similarly, both univariate and multivariate analyses showed that preoperative KPS score and addition of PDT were significant independent prognostic factors for OS (Table 3). In contrast, IDH mutation and pre-recurrence pathology were not significant prognostic factors in this study. 

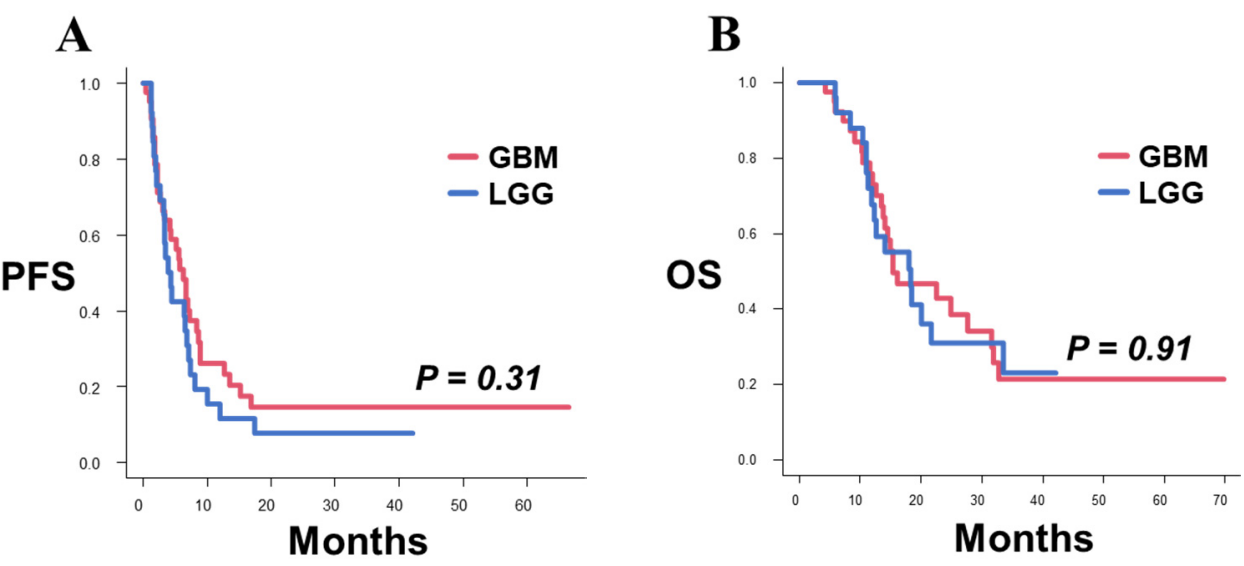

Figure 2. Kaplan-Meier survival curves for PFS and OS after surgery for recurrence in the GBM and LGG groups. (A) The PFS was not significantly different between the GBM and LGG groups (median PFS: GBM 6.3 months, LGG 4.2 months; $p=0.31$ ). (B) The OS was not significantly different between the GBM and LGG groups (median OS: GBM 15.4 months, LGG 18.3 months; $p=0.91$ ).

Table 2. Univariate and multivariate analyses for PFS.

\begin{tabular}{ccccc}
\hline \multirow{2}{*}{ Variables } & & \multicolumn{2}{c}{ Univariate Analysis } & \multicolumn{2}{c}{ Multivariate Analysis } \\
& & Hazard Ratio [95\% CI] & $p$ Value & Hazard Ratio [95\% CI] $p$ Value \\
\hline Age & $<55 y$ vs. $\geq 55 y$ & $1.52[0.95-2.44]$ & 0.079 & $1.82[1.11-2.99]$ \\
\hline KPS & $<70$ vs. $\geq 70$ & $1.70[1.04-2.77]$ & 0.035 & 0.017 \\
\hline Pre-rec pathol & GBM vs. LGG & $0.76[0.51-1.15]$ & 0.2 & 0.13 \\
\hline IDH & mIDH1 vs. wtIDH1 & $1.42[0.91-2.23]$ & 0.005 & $0.52[0.34-0.79]$ \\
\hline PDT & $\begin{array}{c}\text { PDT + Surgery vs. } \\
\text { Surgery alone }\end{array}$ & $0.54[0.35-0.83]$ & & 0.026 \\
\hline
\end{tabular}

Abbreviations: Pre-rec pathol, pre-recurrence pathology; mIDH1, IDH1 mutant type; wtIDH1, IDH1 wild type.

Table 3. Univariate and multivariate analyses for OS.

\begin{tabular}{ccccc}
\hline \multirow{2}{*}{ Variables } & & \multicolumn{2}{c}{ Univariate Analysis } & \multicolumn{2}{c}{ Multivariate Analysis } \\
& & Hazard Ratio [95\% CI] & $p$ Value & Hazard Ratio [95\% CI] $p$ Value \\
\hline Age & $<55 y$ vs. $\geq 55 y$ & $1.36[0.80-2.33]$ & 0.26 & \\
\hline KPS & $<70$ vs. $\geq 70$ & $1.79[1.03-3.09]$ & 0.038 & $1.82[1.05-3.15]$ \\
\hline Pre-rec pathol & GBM vs. LGG & $1.04[0.63-1.69]$ & 0.89 & \\
\hline IDH & mIDH1 vs. wtIDH1 & $1.69[0.98-2.91]$ & 0.06 & \\
\hline PDT & $\begin{array}{c}\text { PDT + Surgery vs. } \\
\text { Surgery alone }\end{array}$ & $0.57[0.34-0.96]$ & 0.034 & $0.56[0.33-0.94]$ \\
\hline
\end{tabular}

\subsection{TS Uptake Comparison}

TS uptake in recurrent GBM and peri-tumoral normal tissue samples was evaluated and photographed using fluorescence microscopy during the surgery (Figure 3). There is greater TS accumulation in recurrent GBM. Among the samples for which intraoperative uptake could be verified, the degree of uptake in seven cases of recurrent GBM was quantitatively compared by classifying them into GBM and LGG groups based on prerecurrence pathology (Figure 4). As a result, 14 samples from five cases in the GBM group and six samples from two cases in the LGG group were analyzed, and there was no significant difference in the fluorescence intensity for each group $(p=0.20)$. 

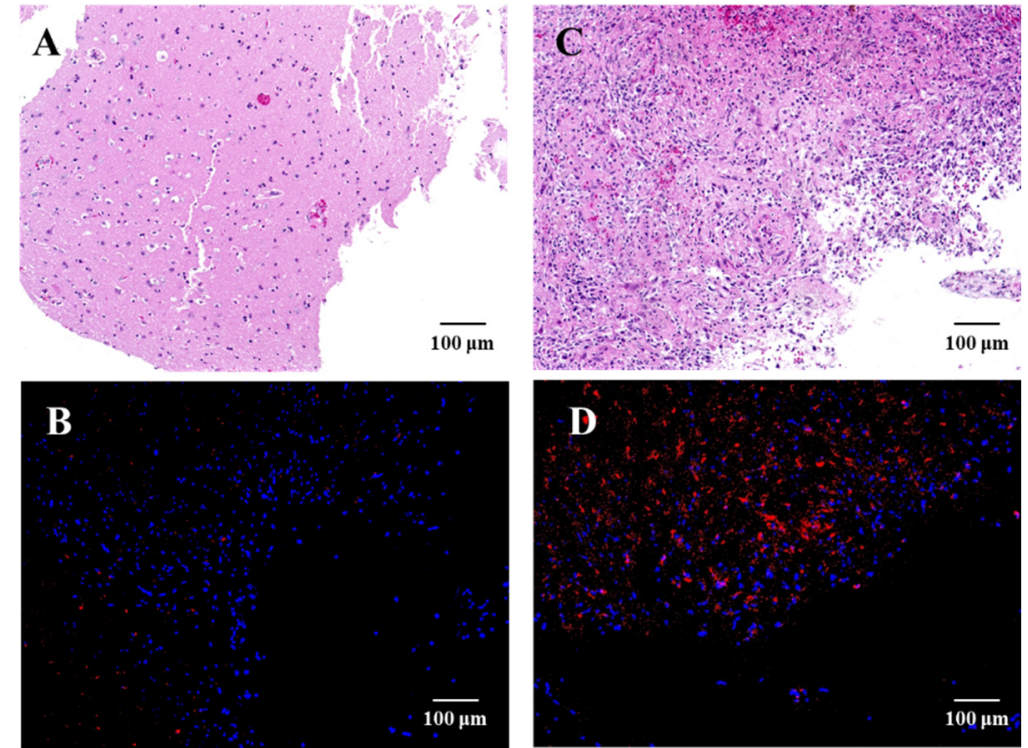

Figure 3. HE staining and fluorescence microscopy images of normal brain tissue surrounding the tumor $(\mathbf{A}, \mathbf{B})$ and recurrent glioblastoma in contrast-enhanced lesions (C,D). Samples were processed for H\&E staining or immunofluorescence examination. The nuclei of the tumor cells were stained with DAPI, and red fluorescence at $640 \mathrm{~nm}$ was detected in the tumor cells at an excitation wavelength of $400 \mathrm{~nm}$, which indicated the TS uptake in the tumor and normal brain tissue. Abbreviations: CE, contrast enhanced.

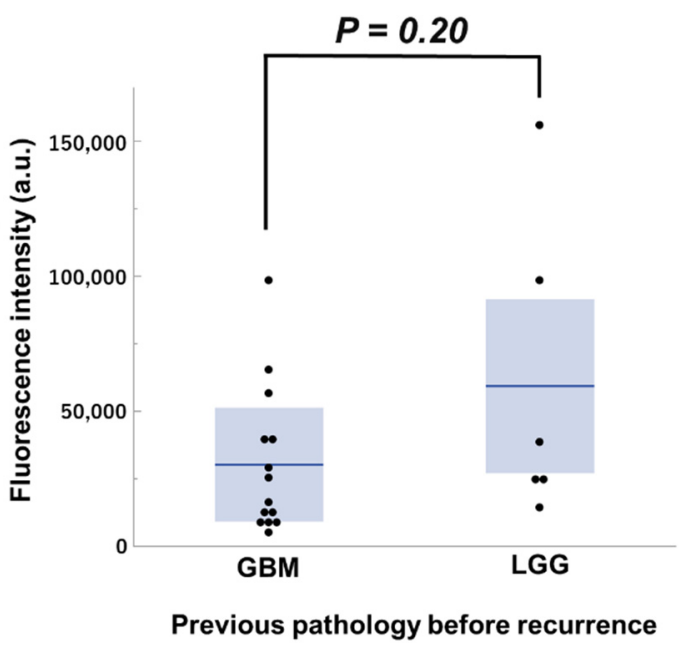

Figure 4. Fluorescence intensity in CE region of recurrent glioblastoma samples from GBM $(n=14)$ and LGG $(n=6)$ groups. There was no significant difference between the two groups (Mann-Whitney $\mathrm{U}$ test, $p=0.20$ ). Each box represents the interquartile range, and the median was indicated by a bold line. The ends of the whiskers represented the 10th and 90th percentile.

\subsection{Representative Cases}

\section{- Case 1}

A 53-year-old woman presented with sudden onset seizure and was hospitalized. MRI revealed a fluid-attenuated inversion recovery (FLAIR) high lesion in the right frontal lobe with no enhancement on gadolinium (Figure 5A,B). Gross-total removal of the FLAIR high lesion was performed via awake craniotomy (Figure 5C,D), and the pathological diagnosis was IDH wildtype anaplastic astrocytoma. The patient received fractionated radiation therapy (60 Gy) and concomitant temozolomide-based chemotherapy followed by maintenance temozolomide-based chemotherapy for another 5 courses. Nine months after the initial operation, follow-up MRI detected a recurrence of the tumor around the 
removal cavity (Figure 5E,F). Gross-total removal of the enhanced lesion was performed, and the cavity wall was irradiated in four spots (Figure $5 \mathrm{G}, \mathrm{H}$ ). The pathological diagnosis was GBM. Postoperatively, the patient transiently exhibited mild manual dexterity and dysphagia. The MRI on day 14 following surgery revealed fluid collection and slight edema (Figure 5I,J), and these findings partially resolved within 2 months (Figure 5K,L). Maintenance temozolomide-based chemotherapy was resumed and continued for 24 courses. At the latest follow-up of 47 months after the second surgery, the MRI demonstrated no recurrence and the patient had a KPS score of 90 (Figure $5 \mathrm{M}, \mathbf{N}$ ).

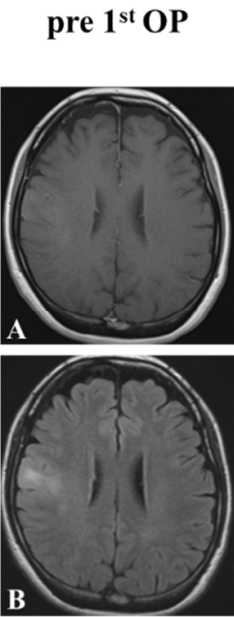

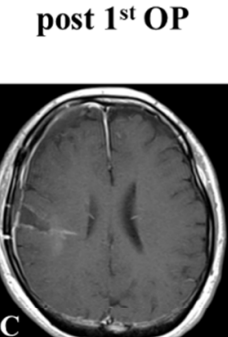

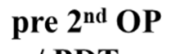

w/ PDT
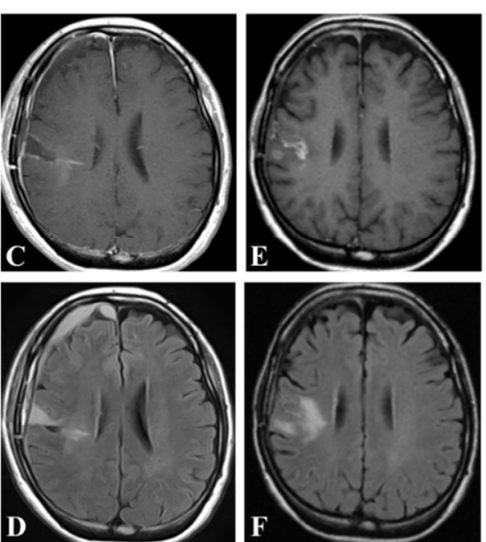

3 days


14 days
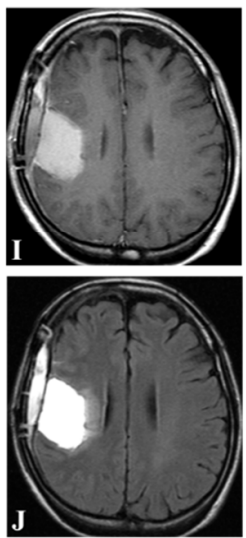

2 months
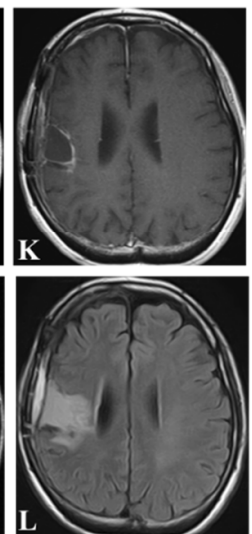

47 months

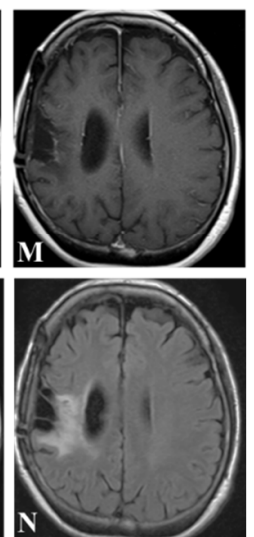

Figure 5. Representative Case 1. Contrast-enhanced T1-weighted and FLAIR MR image before (A,B) and after $(\mathbf{C}, \mathbf{D})$ the first operation. Contrast-enhanced T1-weighted and FLAIR findings before the second operation with PDT (E,F); 3 days $(\mathbf{G}, \mathbf{H}) ; 2$ weeks $(\mathbf{I}, \mathbf{J}) ; 2$ months $(\mathbf{K}, \mathbf{L})$, and 47 months $(\mathbf{M}, \mathbf{N})$ after the second surgery.

\section{- $\quad$ Case 2}

A 59-year-old woman presented with aphasia and speech disturbance. MRI revealed a round mass lesion in the left temporal lobe. The tumor exhibited low intensity on T1weighted images, ring-like enhancement on gadolinium uptake (Figure 6A), and high intensity on FLAIR images (Figure 6B). Gross-total removal of the enhanced lesion was performed (Figure 6C,D), and the pathological diagnosis was IDH wildtype GBM. The patient received fractionated radiation therapy (60 Gy) and concomitant temozolomide-based chemotherapy following autologous formalin-fixed tumor vaccine (AFTV). Three months after the initial operation, follow-up MRI detected tumor recurrence in the anterior part of the removal cavity (Figure 6E,F). Gross-total removal of the enhanced lesion was performed again, and the cavity wall was irradiated in six spots (Figure 6G,H). The pathological diagnosis was GBM. Postoperatively, the patient still exhibited mild aphasia; the symptoms were unchanged compared to before the surgery. The 14-day postoperative MRI revealed fluid collection and edema (Figure 6I,J), and these resolved within 2 months (Figure 6K,L). Five months after the second operation, an enhanced lesion appeared in the medial part of the cavity wall (Figure 6M), and it exhibited uptake in a methionine PET study with a tumor tissue/normal tissue ratio of 2.74 (Figure $6 \mathrm{~N}$ ). Removal of the enhanced lesion was performed, and the cavity wall was irradiated again in six spots. The pathological diagnosis was necrotic tissue, and there was no evidence of tumor recurrence (Figure 6Q,R). At the latest follow-up, 50 months after the second surgery, the MRI showed no recurrence and the patient had a KPS score of 80 (Figure 6O,P). 

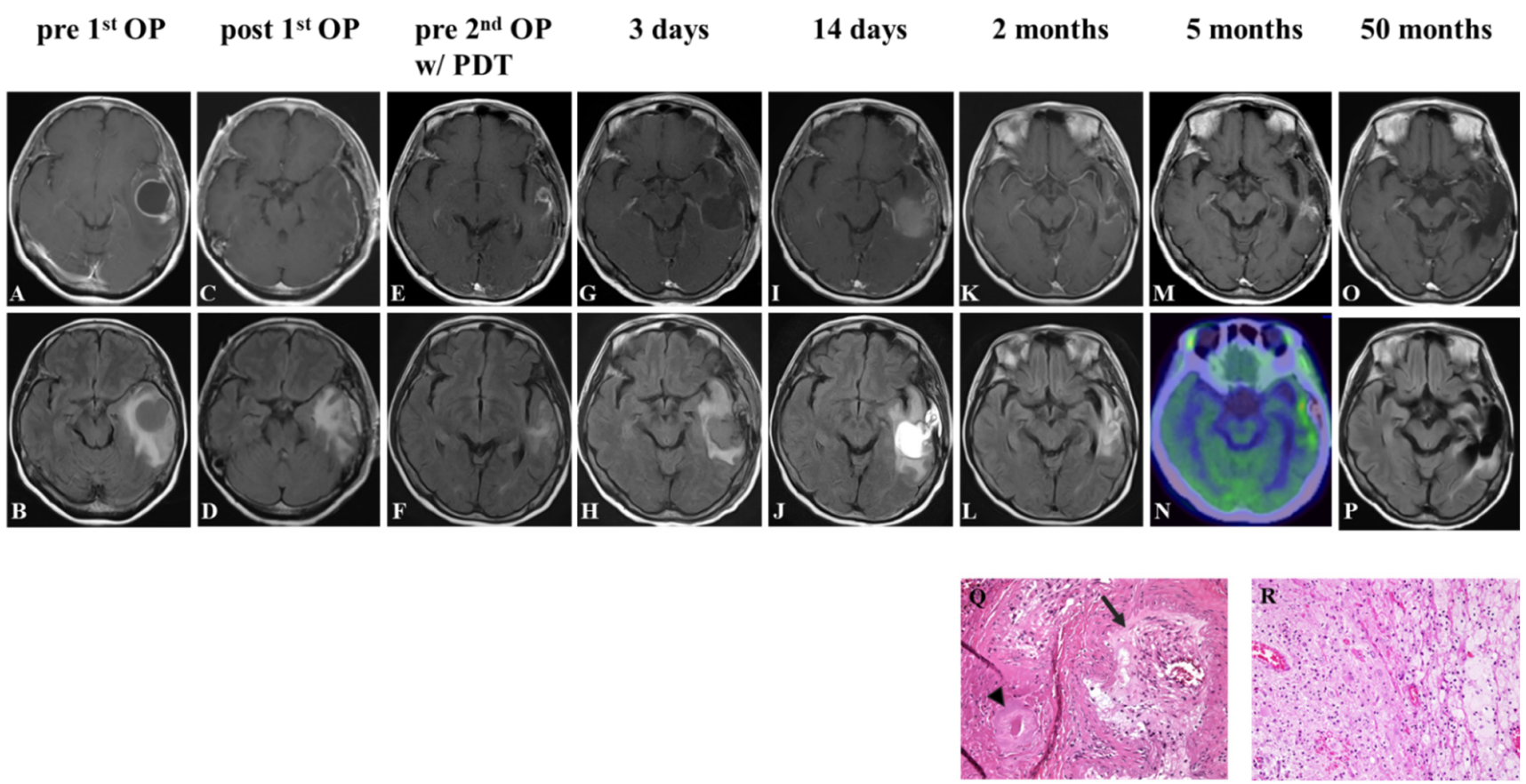

Figure 6. Representative Case 2. Contrast-enhanced T1-weighted and FLAIR MR image before (A,B) and after $(\mathbf{C}, \mathbf{D})$ the first operation. Contrast-enhanced T1-weighted and FLAIR findings before the second operation with PDT (E,F); 3 days $(\mathbf{G}, \mathbf{H}) ; 2$ weeks $(\mathbf{I}, \mathbf{J}) ; 2$ months $(\mathbf{K}, \mathbf{L}) ; 5$ months $(\mathbf{M})$, and 50 months $(\mathbf{O}, \mathbf{P})$ after the second surgery. PET study at 5 months postoperatively $(\mathbf{N})$. The pathological results of the suspected recurrent lesion at 5 months postoperatively showed venous infarction $((\mathbf{Q})$ arrowhead) and loss of internal elastic membrane $((\mathbf{Q})$ arrow), but no obvious tumor cells (R).

\section{Discussion}

Although the first uses of light as a therapeutic agent date back many centuries, the evolution of PDT as a cancer treatment occurred 1940-1950 with the discovery and purification of hematoporphyrin derivatives, which were highly accumulative in cancerous tissue [21]. Since then, a large number of photosensitizers have been developed for PDT against tumors [22]. To date, studies of PDT on tumors have shown that the mechanism of tumor cell death and destruction varies depending on the type of photosensitizer and the irradiation conditions including a combination of apoptosis, necrosis, autophagy, necroptosis, parthanatos or other regulated cell death, immunogenic cell death, and cell death due to microvascular damage or occlusion [23-27]. In addition to brain tumors, several studies on photosensitizers have demonstrated efficacy in various cancers such as lung, esophageal, head and neck, otorhinolaryngological, skin, hepatobiliary, pancreatic, colorectal, prostate, bladder, and ovarian cancers $[9,28]$. Despite several potential candidates as photosensitizers for use in the treatment of brain tumors, a limited number are currently being used clinically based on the results of clinical trials and side effects such as photosensitivity $[29,30]$. In recent years, the photosensitizers used in clinical trials for malignant brain tumors are mainly TS [31] and 5-ALA [32-34], both of which exhibit a favorable safety profile.

Tumor accumulation rate is known to vary depending on the photosensitizer, and previous in vivo experiments demonstrated that the uptake level of TS in the brain tumor was 23.1 times that of normal brain tissue, which was also 7.78 times higher than the uptake of 5-ALA and 13 times higher than that of Photofrin [35,36]. Therefore, TS-PDT was expected to be a useful additional treatment for malignant brain tumors. In fact, a previously reported comparative retrospective analysis of surgical resection with TS-PDT in 30 consecutive cases of newly diagnosed GBM and 164 consecutive cases treated with surgical resection alone during the same period showed a satisfactory result [13]. Contrarily, 
while the efficacy of TS-PDT in recurrent malignant brain tumors is an important clinical question, there has only been one clinical report in a limited number of patients [14].

In this study, we focused on recurrent GBM and evaluated the efficacy of TS-PDT. As shown in the time-to-event analysis, the addition of TS-PDT to surgical treatment in recurrent GBM significantly improved the prognosis compared with surgical treatment alone and the efficacy of TS-PDT was independent of the pre-recurrence pathology. The PFS curve of the PDT group decreased relatively early; subsequently, a certain number of patients exhibited long-term PFS. We previously reported that tumors can exhibit malignant behavior at the cellular level when the effect of TS-PDT is inadequate [26]; the early decline in the PFS curve may be due to inadequate TS-PDT effect, and a certain number of patients with long-term PFS may be due to adequate antitumor effect of TS-PDT. Although the factors that make TS-PDT effective are unclear, the difference in TS-PDT efficacy despite most patients achieving GTR may be related to its effect on tumors lodged in the FLAIR high region or secondary effects such as antitumor immunity [37].

The results of univariate and multivariate analyses suggested that the addition of TS-PDT might be a prognostic factor along with the preoperative KPS, which is a known prognostic factor in recurrent GBM. However, IDH mutation and the pre-recurrence pathology were not prognostic factors. In particular, IDH mutation is known to be a significant prognostic factor in newly diagnosed GBM [38], but its clinical importance in recurrent GBM remains controversial. In the literature on recurrent malignant glioma, there are reports that IDH mutation was associated with a better prognosis [39], whereas there are also reports of better results with IDH wildtype malignant glioma with respect to combined stereotactic radiotherapy with bevacizumab [4]. Contrarily, several publications have reported that IDH mutation is not a prognostic factor at the time of recurrent GBM [40,41], and our results are in agreement with these reports. In view of previous reports and our study results, IDH mutation is a strong prognostic marker of glioma characteristics during the early stage, but it may lose its pivotal role as a prognostic factor when the tumor recurs and pathologically develops as GBM. Taken together, the results of the time-to-event analysis and multivariate analysis suggest that there is benefit in adding TS-PDT to tumor resection when the recurrent tumor is suspected to be a GBM, regardless of its origin or molecular biology.

When photosensitizers accumulate in tumors, peritumoral vessels, or peritumoral tissue stroma, there is debate about which localization determines the impact of PDT's antitumor effects. Although this has not been fully elucidated in TS-PDT in the brain tumor environment, reports using TS-PDT for tumors in other cancers or using other photosensitizers suggest that accumulation of TS in the tumor itself strongly influences the antitumor effects of TS-PDT, as much as accumulation in peritumor blood vessels [42-44]. Our results showed that intra-tumoral accumulation of TS was independent of the prerecurrence pathology. This result may support that the clinical efficacy of additional PDT for recurrent GBM observed in this study was independent of the patient's previous pathology.

Concerning the effect of PDT as shown in this study, innovations in drug delivery such as the accumulation of photosensitizers in tumors and peritumoral blood vessels are expected to further enhance this anti-tumor effect, and novel research is being conducted regarding various methods. For example, there are reports on the enhancement of tumor accumulation and antitumor effect by conjugating photosensitizers with various nanoparticles [45], conjugating with tumor-specific antibodies [46], and PDT using a multi-targeted liposome system in which photosensitizers are encapsulated in liposomes targeting the tumor, vascular endothelium, and tumor stroma [47]. With regard to TS, efforts to improve tumor accumulation by incorporating TS into inactivated viral envelopes have recently been reported [48]. The antitumor effect of TS-PDT for recurrent GBM demonstrated in this study may be further improved by devising such a drug-delivery system, and it is expected to evolve as a therapeutic method in the future.

The primary limitation of this study was its retrospective nature. The results of this study were subject to selection bias since all patients underwent surgery for a recurrent 
lesion. Only recurrent lesions considered removable were eligible for surgery at our institution. Therefore, cases in which surgical removal was difficult due to eloquent lesions or multiple lesions, were excluded.

Despite this limitation, we believe that this study, which demonstrated the efficacy of TS-PDT in treating recurrent GBM, is very valuable with potential clinical application. Future prospective clinical trials are required to confirm the results of this study.

\section{Conclusions}

TS-PDT has significant prognostic value as an adjunct to surgery in treating recurrent GBM. This effect is independent of pre-recurrence pathology and is a versatile additional treatment.

Author Contributions: Conceptualization, T.K. (Tatsuya Kobayashi), M.N. and Y.M.; methodology, T.K. (Tatsuya Kobayashi) and M.N.; formal analysis, T.K. (Tatsuya Kobayashi) and K.S.; investigation, T.K. (Tatsuya Kobayashi), M.N. and K.S.; Resources, T.K. (Tatsuya Kobayashi), K.S., T.S., S.T., A.F., S.K., A.K., T.K. (Takashi Komori) and K.M.; writing—original draft preparation, T.K. (Tatsuya Kobayashi); writing-review and editing, M.N., T.S., T.K. (Takashi Komori), T.M., T.K. (Takakazu Kawamata) and Y.M.; visualization, T.K. (Tatsuya Kobayashi) and K.S.; supervision, M.N., T.M, T.K. (Takakazu Kawamata) and Y.M; project administration, T.K. (Tatsuya Kobayashi), M.N. and Y.M. All authors have read and agreed to the published version of the manuscript.

Funding: This research received no external funding.

Institutional Review Board Statement: This study was conducted according to the guidelines of the Declaration of Helsinki, and approved by the Institutional Review Board of Tokyo Women's Medical University (approval no.: 3540-R6, approved 17 July 2019).

Informed Consent Statement: Patient consent was waived due to the retrospective design of the study.

Data Availability Statement: The data presented in this study are available on request from the corresponding author.

Acknowledgments: We thank Takashi Sakayori and Asuka Komori (Tokyo Women's Medical University) for their kind support in the neuropathological and pharmacokinetic evaluation.

Conflicts of Interest: The authors declare no conflict of interest.

\section{References}

1. Stupp, R.; Mason, W.P.; van den Bent, M.J.; Weller, M.; Fisher, B.; Taphoorn, M.J.B.; Belanger, K.; Brandes, A.A.; Marosi, C.; Bogdahn, U.; et al. Radiotherapy plus Concomitant and Adjuvant Temozolomide for Glioblastoma. N. Engl. J. Med. 2005, 352, 987-996. [CrossRef] [PubMed]

2. Wong, E.T.; Hess, K.R.; Gleason, M.J.; Jaeckle, K.A.; Kyritsis, A.P.; Prados, M.D.; Levin, V.A.; Yung, W.K. Outcomes and prognostic factors in recurrent glioma patients enrolled onto phase II clinical trials. J. Clin. Oncol. Off. J. Am. Soc. Clin. Oncol. 1999, 17, 2572-2578. [CrossRef] [PubMed]

3. $\quad$ van Linde, M.E.; Brahm, C.G.; de Witt Hamer, P.C.; Reijneveld, J.C.; Bruynzeel, A.M.E.; Vandertop, W.P.; van de Ven, P.M.; Wagemakers, M.; van der Weide, H.L.; Enting, R.H.; et al. Treatment outcome of patients with recurrent glioblastoma multiforme: A retrospective multicenter analysis. J. Neurooncol. 2017, 135, 183-192. [CrossRef] [PubMed]

4. Yasuda, T.; Muragaki, Y.; Nitta, M.; Miyamoto, K.; Oura, Y.; Henmi, T.; Noguchi, S.; Oda, H.; Saito, T.; Maruyama, T.; et al. Effectiveness of Stereotactic Radiotherapy and Bevacizumab for Recurrent High-Grade Gliomas: A Potential Therapy for Isocitrate Dehydrogenase Wild-Type Recurrent High-Grade Gliomas. World Neurosurg. 2018, 114, e1138-e1146. [CrossRef] [PubMed]

5. Di Nunno, V.; Franceschi, E.; Tosoni, A.; Di Battista, M.; Gatto, L.; Lamperini, C.; Minichillo, S.; Mura, A.; Bartolini, S.; Brandes, A.A. Treatment of recurrent glioblastoma: State-of-the-art and future perspectives. Expert Rev. Anticancer Ther. 2020, $20,785-795$. [CrossRef] [PubMed]

6. Kamiya-Matsuoka, C.; Gilbert, M.R. Treating recurrent glioblastoma: An update. CNS Oncol. 2015, 4, 91-104. [CrossRef] [PubMed]

7. Kwiatkowski, S.; Knap, B.; Przystupski, D.; Saczko, J.; Kędzierska, E.; Knap-Czop, K.; Kotlińska, J.; Michel, O.; Kotowski, K.; Kulbacka, J. Photodynamic therapy-Mechanisms, photosensitizers and combinations. Biomed. Pharmacother. 2018, 106, 1098-1107. [CrossRef] [PubMed]

8. Castano, A.P.; Demidova, T.N.; Hamblin, M.R. Mechanisms in photodynamic therapy: Part one-Photosensitizers, photochemistry and cellular localization. Photodiagn. Photodyn. Ther. 2004, 1, 279-293. [CrossRef] 
9. van Straten, D.; Mashayekhi, V.; de Bruijn, H.S.; Oliveira, S.; Robinson, D.J. Oncologic photodynamic therapy: Basic principles, current clinical status and future directions. Cancers 2017, 9, 19. [CrossRef] [PubMed]

10. Kato, H.; Furukawa, K.; Sato, M.; Okunaka, T.; Kusunoki, Y.; Kawahara, M.; Fukuoka, M.; Miyazawa, T.; Yana, T.; Matsui, K.; et al Phase II clinical study of photodynamic therapy using mono-L-aspartyl chlorin e6 and diode laser for early superficial squamous cell carcinoma of the lung. Lung Cancer 2003, 42, 103-111. [CrossRef]

11. Yano, T.; Kasai, H.; Horimatsu, T.; Yoshimura, K.; Teramukai, S.; Morita, S.; Tada, H.; Yamamoto, Y.; Kataoka, H.; Kakushima, N.; et al. A multicenter phase II study of salvage photodynamic therapy using talaporfin sodium (ME2906) and a diode laser (PNL6405EPG) for local failure after chemoradiotherapy or radiotherapy for esophageal cancer. Oncotarget 2017, 8, 22135-22144. [CrossRef]

12. Muragaki, Y.; Akimoto, J.; Maruyama, T.; Iseki, H.; Ikuta, S.; Nitta, M.; Maebayashi, K.; Saito, T.; Okada, Y.; Kaneko, S.; et al. Phase II clinical study on intraoperative photodynamic therapy with talaporfin sodium and semiconductor laser in patients with malignant brain tumors. J. Neurosurg. 2013, 119, 845-852. [CrossRef]

13. Nitta, M.; Muragaki, Y.; Maruyama, T.; Iseki, H.; Komori, T.; Ikuta, S.; Saito, T.; Yasuda, T.; Hosono, J.; Okamoto, S.; et al. Role of photodynamic therapy using talaporfin sodium and a semiconductor laser in patients with newly diagnosed glioblastoma. $J$. Neurosurg. 2019, 131, 1361-1368. [CrossRef]

14. Akimoto, J.; Haraoka, J.; Aizawa, K. Preliminary clinical report on safety and efficacy of photodynamic therapy using talaporfin sodium for malignant gliomas. Photodiagn. Photodyn. Ther. 2012, 9, 91-99. [CrossRef]

15. Louis, D.N.; Ohgaki, H.; Wiestler, O.D.; Cavenee, W.K.; Burger, P.C.; Jouvet, A. The 2007 WHO classification of tumours of the central nervous system. Acta Neuropathol. 2007, 114, 97-109. [CrossRef]

16. Louis, D.N.; Perry, A.; Reifenberger, G.; von Deimling, A.; Figarella-Branger, D.; Cavenee, W.K.; Ohgaki, H.; Wiestler, O.D.; Kleihues, P.; Ellison, D.W. The 2016 World Health Organization Classification of Tumors of the Central Nervous System: A summary. Acta Neuropathol. 2016, 131, 803-820. [CrossRef]

17. Aizawa, K.; Okunaka, T.; Ohtani, T.; Kawabe, H.; Yasunaka, Y.; O’Hata, S.; Ohtomo, N.; Nishimiya, K.; Konaka, C.; Kato, H.; et al Localization of mono-L-aspartyl chlorin e6 (NPe6) in mouse tissues. Photochem. Photobiol. 1987, 46, 789-793. [CrossRef]

18. Shimizu, K.; Nitta, M.; Komori, T.; Maruyama, T.; Yasuda, T.; Fujii, Y.; Masamune, K.; Kawamata, T.; Maehara, T.; Muragaki, Y. Intraoperative photodynamic diagnosis using talaporfin sodium simultaneously applied for photodynamic therapy against malignant glioma: A prospective clinical study. Front. Neurol. 2018, 9, 1-9. [CrossRef]

19. Wen, P.Y.; Macdonald, D.R.; Reardon, D.A.; Cloughesy, T.F.; Sorensen, A.G.; Galanis, E.; Degroot, J.; Wick, W.; Gilbert, M.R.; Lassman, A.B.; et al. Updated response assessment criteria for high-grade gliomas: Response assessment in neuro-oncology working group. J. Clin. Oncol. Off. J. Am. Soc. Clin. Oncol. 2010, 28, 1963-1972. [CrossRef]

20. Kanda, Y. Investigation of the freely available easy-to-use software 'EZR' for medical statistics. Bone Marrow Transplant. 2013, 48, 452-458. [CrossRef]

21. Daniell, M.D.; Hill, J.S. A history of photodynamic therapy. Aust. N. Z. J. Surg. 1991, 61, 340-348. [CrossRef] [PubMed]

22. Ormond, A.B.; Freeman, H.S. Dye sensitizers for photodynamic therapy. Materials 2013, 6, 817-840. [CrossRef] [PubMed]

23. Yoo, J.O.; Ha, K.S. New Insights into the Mechanisms for Photodynamic Therapy-Induced Cancer Cell Death, 1st ed.; Elsevier Inc.: Amsterdam, The Netherlands, 2012; Volume 295, ISBN 9780123943064.

24. Garg, A.D.; Maes, H.; Romano, E.; Agostinis, P. Autophagy, a major adaptation pathway shaping cancer cell death and anticancer immunity responses following photodynamic therapy. Photochem. Photobiol. Sci. 2015, 14, 1410-1424. [CrossRef] [PubMed]

25. Soriano, J.; Mora-Espí, I.; Alea-Reyes, M.E.; Pérez-García, L.; Barrios, L.; Ibáñez, E.; Nogués, C. Cell death mechanisms in Tumoral and Non-Tumoral human cell lines triggered by photodynamic treatments: Apoptosis, necrosis and parthanatos. Sci. Rep. 2017, 7, 1-13. [CrossRef] [PubMed]

26. Kobayashi, T.; Miyazaki, M.; Sasaki, N.; Yamamuro, S.; Uchida, E.; Kawauchi, D.; Takahashi, M.; Otsuka, Y.; Kumagai, K.; Takeuchi, S.; et al. Enhanced Malignant Phenotypes of Glioblastoma Cells Surviving NPe6-Mediated Photodynamic Therapy are Regulated via ERK1/2 Activation. Cancers 2020, 12, 3641. [CrossRef] [PubMed]

27. Sirotkina, M.A.; Matveev, L.A.; Shirmanova, M.V.; Zaitsev, V.Y.; Buyanova, N.L.; Elagin, V.V.; Gelikonov, G.V.; Kuznetsov, S.S.; Kiseleva, E.B.; Moiseev, A.A.; et al. Photodynamic therapy monitoring with optical coherence angiography. Sci. Rep. 2017, 7, 1-11. [CrossRef] [PubMed]

28. Agostinis, P.; Berg, K.; Cengel, K.A.; Foster, T.H.; Girotti, A.W.; Gollnick, S.O.; Hahn, S.M.; Hamblin, M.R.; Juzeniene, A.; Kessel, D.; et al. Photodynamic therapy of cancer: An update. CA Cancer J. Clin. 2011, 61, 250-281. [CrossRef]

29. Cramer, S.W.; Chen, C.C. Photodynamic Therapy for the Treatment of Glioblastoma. Front. Surg. 2020, 6, 1-11. [CrossRef]

30. Quirk, B.J.; Brandal, G.; Donlon, S.; Vera, J.C.; Mang, T.S.; Foy, A.B.; Lew, S.M.; Girotti, A.W.; Jogal, S.; LaViolette, P.S.; et al. Photodynamic therapy (PDT) for malignant brain tumors-Where do we stand? Photodiagn. Photodyn. Ther. 2015, 12, 530-544. [CrossRef] [PubMed]

31. Japan Registry of Clinical Trials. Available online: https://jrct.niph.go.jp/en-latest-detail/jRCTs031180360 (accessed on 25 July 2021)

32. German Clinical Trials Register. Available online: https://www.drks.de/drks_web/navigate.do?navigationId=trial.HTML\& TRIAL_ID=DRKS00020543 (accessed on 25 July 2021).

33. Aktuelle Studien IStudien I NOA-Neuroonkologische Arbeitsgemeinschaft. Available online: https://www.neuroonkologie.de/ studien (accessed on 25 July 2021). 
34. Vermandel, M.; Dupont, C.; Lecomte, F.; Leroy, H.A.; Tuleasca, C.; Mordon, S.; Hadjipanayis, C.G.; Reyns, N. Standardized intraoperative 5-ALA photodynamic therapy for newly diagnosed glioblastoma patients: A preliminary analysis of the INDYGO clinical trial. J. Neurooncol. 2021, 152, 501-514. [CrossRef]

35. Tsurubuchi, T.; Zoboronok, A.; Yamamoto, T.; Nakai, K.; Yoshida, F.; Shirakawa, M.; Matsuda, M.; Matsumura, A. The optimization of fluorescence imaging of brain tumor tissue differentiated from brain edema-In vivo kinetic study of 5-aminolevulinic acid and talaporfin sodium. Photodiagn. Photodyn. Ther. 2009, 6, 19-27. [CrossRef]

36. Chopp, M.; Dereski, M.O.; Madigan, L.; Jiang, F.; Logie, B. Sensitivity of 9L gliosarcomas to photodynamic therapy. Radiat. Res. 1996, 146, 461-465. [CrossRef]

37. Gollnick, S.O. Photodynamic therapy and antitumor immunity. J. Natl. Compr. Canc. Netw. 2012, 10 (Suppl. 2), S40-S43. [CrossRef]

38. Sanson, M.; Marie, Y.; Paris, S.; Idbaih, A.; Laffaire, J.; Ducray, F.; El Hallani, S.; Boisselier, B.; Mokhtari, K.; Hoang-Xuan, K.; et al. Isocitrate dehydrogenase 1 codon 132 mutation is an important prognostic biomarker in gliomas. J. Clin. Oncol. 2009, 27, 4150-4154. [CrossRef]

39. Collins, V.P.; Ichimura, K.; Di, Y.; Pearson, D.; Chan, R.; Thompson, L.C.; Gabe, R.; Brada, M.; Stenning, S.P. Prognostic and predictive markers in recurrent high grade glioma; results from the BR12 randomised trial. Acta Neuropathol. Commun. 2014, 2, 1-15. [CrossRef]

40. Mandel, J.J.; Cachia, D.; Liu, D.; Wilson, C.; Aldape, K.; Fuller, G.; de Groot, J.F. Impact of IDH1 mutation status on outcome in clinical trials for recurrent glioblastoma. J. Neurooncol. 2016, 129, 147-154. [CrossRef]

41. Tabei, Y.; Kobayashi, K.; Saito, K.; Shimizu, S.; Suzuki, K.; Sasaki, N.; Shiokawa, Y.; Nagane, M. Survival in patients with glioblastoma at a first progression does not correlate with isocitrate dehydrogenase (IDH)1 gene mutation status. Jpn. J. Clin. Oncol. 2021, 51, 45-53. [CrossRef]

42. McMahon, K.S.; Wieman, T.J.; Moore, P.H.; Fingar, V.H. Effects of photodynamic therapy using mono-L-aspartyl chlorin e6 on vessel constriction, vessel leakage, and tumor response. Cancer Res. 1994, 54, 5374-5379.

43. Jones, H.J.; Vernon, D.I.; Brown, S.B. Photodynamic therapy effect of m-THPC (Foscan) in vivo: Correlation with pharmacokinetics. Br. J. Cancer 2003, 89, 398-404. [CrossRef]

44. Du, H.-Y.; Bay, B.-H.; Olivo, M. Biodistribution and photodynamic therapy with hypericin in a human NPC murine tumor model. Int. J. Oncol. 2003, 22, 1019-1024. [CrossRef]

45. Alsaab, H.O.; Alghamdi, M.S.; Alotaibi, A.S.; Alzhrani, R.; Alwuthaynani, F.; Althobaiti, Y.S.; Almalki, A.H.; Sau, S.; Iyer, A.K. Progress in Clinical Trials of Photodynamic Therapy for Solid Tumors and the Role of Nanomedicine. Cancers 2020, $12,2793$. [CrossRef] [PubMed]

46. Kobayashi, H.; Griffiths, G.L.; Choyke, P.L. Near-Infrared Photoimmunotherapy: Photoactivatable Antibody-Drug Conjugates (ADCs). Bioconjug. Chem. 2020, 31, 28-36. [CrossRef] [PubMed]

47. Weijer, R.; Broekgaarden, M.; Kos, M.; van Vught, R.; Rauws, E.A.J.; Breukink, E.; van Gulik, T.M.; Storm, G.; Heger, M. Enhancing photodynamic therapy of refractory solid cancers: Combining second-generation photosensitizers with multi-targeted liposomal delivery. J. Photochem. Photobiol. C Photochem. Rev. 2015, 23, 103-131. [CrossRef]

48. Akter, S.; Inai, M.; Saito, S.; Honda, N.; Hazama, H.; Nishikawa, T.; Kaneda, Y.; Awazu, K. Photodynamic therapy by lysosomaltargeted drug delivery using talaporfin sodium incorporated into inactivated virus particles. Laser Ther. 2019, 28, $245-256$. [CrossRef] 